hep-ph/9807283v2

EHU-07 Revised, September 1998

\title{
Vacuum Neutrino Oscillations of Solar Neutrinos and Lepton Mass Matrices
}

\author{
Morimitsu TANIMOTOQ \\ Science Education Laboratory, Ehime University, 790-8577 Matsuyama, JAPAN
}

\begin{abstract}
We consider the case that the solar neutrino deficit is due to the vacuum oscillation. The lepton mass matrices with nearly bi-maximal mixings are needed in order to explain both solar and atmospheric neutrino deficit. A texture with the symmetry of flavour democracy or $S_{3}$ has been investigated by taking account of the symmetry breaking terms of the charged lepton mass matrix. It is found that predicted mixings can be considerably changed from the neutrino mixings $\sin ^{2} 2 \theta_{\odot} \simeq 1$ and $\sin ^{2} 2 \theta_{\text {atm }} \simeq 8 / 9$ at the symmetric limit. The correlation between $\left|U_{e 3}\right|$ and $\left|U_{e 1} U_{e 2}^{*}\right|$ is also presented. The test of the model is discussed by focusing on the three flavor analyses in the solar neutrinos, atmospheric neutrinos and long baseline experiments.
\end{abstract}

\footnotetext{
${ }^{1}$ E-mail address: tanimoto@edserv.ed.ehime-u.ac.jp
} 
Neutrino flavor oscillations provide information of the fundamental property of neutrinos such as masses, flavor mixings. In these years, there is growing experimental evidences of neutrino oscillations. The exciting one is the atmospheric neutrino deficit [四 [3] as well as the solar neutrino deficit [4. Super-Kamiokande [5] presented the near-maximal neutrino flavor oscillation in atmospheric neutrinos. Recent Super-Kamiokande data also suggest that vacuum oscillation regions are favored in the analysis of day-night spectra and energy shape of the solar neutrino [6] $[8]$. Therefore, three flavor analyses are very important for the vacuum oscillation of solar neutrinos [9] [10]. Results of those analyses can give constraints on the structure of the lepton mass matrices. Therefore, it is urgent to make clear predictions quantitatively in the lepton mass matrix models which give near-maximal solar and atmospheric vacuum oscillations [11] 13].

There is a typical texture of the lepton mass matrix with nearly bi-maximal mixing of three neutrinos, which is derived from the symmetry of the lepton flavor democracy by Fritzsch and Xing [14], or from the $S_{3 L} \times S_{3 R}$ symmetry of the left-handed Majorana neutrino mass matrix given by Fukugita, Tanimoto and Yanagida [15]. These models can give identical predictions for the neutrino mixings $\sin ^{2} 2 \theta_{\odot} \simeq 1$ and $\sin ^{2} 2 \theta_{\text {atm }} \simeq 8 / 9$ although those depend on the symmetry breaking patterns. However, these predictions are considerably changed if the symmetry breaking terms are correctly taking into account. In this paper, we investigate the quantitative predictions on the neutrino flavor mixings including symmetry breaking terms, which become significant for long baseline(LBL) neutrino oscillation experiments. In particular, LBL experiments of $\nu_{\mu} \rightarrow \nu_{e}$ can test the prediction of $U_{e 3}$ in the model. The first LBL reactor experiment CHOOZ has already reported a bound of the neutrino oscillation [16], which gives a strong constraint of the flavor mixing pattern. The LBL accelerator experiment K2K [17] is planned to begin taking data in the next year, whereas the MINOS [18] and ICARUS [19] experiments will start in the first year of the next century. Thus, the 
lepton mass matrix model will be tested in the near future.

Our conservative approach is to assume that oscillations need only account for the solar and atmospheric neutrino data. Since the result of LSND [20] awaits confirmation by KARMEN experiment 21], we do not take into consideration the LSND data in this paper. Recent results of atmospheric neutrinos at Super-Kamiokande [5] suggest $\nu_{\mu} \rightarrow \nu_{\tau}$ oscillation with the near-maximal mixing. Since the CHOOZ result 16 excludes the large neutrino oscillation of $\nu_{\mu} \rightarrow \nu_{e}$ as far as $\Delta m^{2} \geq 9 \times 10^{-4} \mathrm{eV}^{2}$, the large mixing between $\nu_{\mu}$ and $\nu_{\tau}$ is a reasonable interpretation for the atmospheric $\nu_{\mu}$ deficit. Our starting point as to neutrino mixings is the near-maximal $\nu_{\mu} \rightarrow \nu_{\tau}$ oscillation with

$$
\Delta m_{\mathrm{atm}}^{2}=10^{-3} \sim 10^{-2} \mathrm{eV}^{2}, \quad \sin ^{2} 2 \theta_{\mathrm{atm}} \geq 0.8,
$$

which constrain neutrino oscillations in LBL experiments. On the other hand, recent solar neutrino data of Super-Kamiokande [6] favors vacuum long-wavelength oscillations with $\Delta m_{\odot}^{2} \simeq 10^{-10} \mathrm{eV}^{2}$ and the near-maximal mixing in the analysis of day-night spectra and energy shape.

The texture of the lepton mass matrices with nearly bi-maximal mixing of three neutrinos was presented based on the democratic mass matrix as follows [14][15]: the charged lepton mass matrix is

$$
M_{\ell}=\frac{c_{\ell}}{3}\left(\begin{array}{ccc}
1 & 1 & 1 \\
1 & 1 & 1 \\
1 & 1 & 1
\end{array}\right)+\left(\begin{array}{ccc}
\delta_{\ell} & 0 & 0 \\
0 & \rho_{\ell} & 0 \\
0 & 0 & \epsilon_{\ell}
\end{array}\right),
$$

where the second matrix is the symmetry breaking terms, which were given for quark mass matrices by Koide [22], and $\delta_{\ell}, \rho_{\ell}$ and $\epsilon_{\ell}$ are complex parameters in general. By neglecting $C P$ violating phases and using $c_{\ell} \gg \epsilon_{\ell} \gg \rho_{\ell}, \delta_{\ell}$, this matrix is diagonalized approximately as

$$
V_{\ell}^{\dagger} M_{\ell} V_{\ell}=\operatorname{diag}\left(m_{\ell 1}, m_{\ell 2}, m_{\ell 3}\right)
$$


where

$$
\begin{aligned}
& m_{\ell 1}=\left(\delta_{\ell}+\rho_{\ell}+\epsilon_{\ell}\right) / 3-\xi_{\ell} / 6, \\
& m_{\ell 2}=\left(\delta_{\ell}+\rho_{\ell}+\epsilon_{\ell}\right) / 3+\xi_{\ell} / 6, \\
& m_{\ell 3}=c_{\ell}+\left(\delta_{\ell}+\rho_{\ell}+\epsilon_{\ell}\right) / 3,
\end{aligned}
$$

with

$$
\xi_{\ell}=\left[\left(2 \epsilon_{\ell}-\rho_{\ell}-\delta_{\ell}\right)^{2}+3\left(\rho_{\ell}-\epsilon_{\ell}\right)^{2}\right]^{1 / 2}
$$

The unitary matrix $V_{\ell}$ is given as $V_{\ell}=F L$, where

$$
\begin{aligned}
F & =\left(\begin{array}{ccc}
1 / \sqrt{2} & 1 / \sqrt{6} & 1 / \sqrt{3} \\
-1 / \sqrt{2} & 1 / \sqrt{6} & 1 / \sqrt{3} \\
0 & -2 / \sqrt{6} & 1 / \sqrt{3}
\end{array}\right), \\
L \simeq & \left(\begin{array}{ccc}
\cos \theta_{\ell} & -\sin \theta_{\ell} & \lambda_{\ell} \sin 2 \theta_{\ell} \\
\sin \theta_{\ell} & \cos \theta_{\ell} & \lambda_{\ell} \cos 2 \theta_{\ell} \\
-\lambda_{\ell} \sin 3 \theta_{\ell} & -\lambda_{\ell} \cos 3 \theta_{\ell} & 1
\end{array}\right),
\end{aligned}
$$

with

$$
\tan 2 \theta_{\ell} \simeq-\sqrt{3} \frac{\rho_{\ell}-\delta_{\ell}}{2 \epsilon_{\ell}-\rho_{\ell}-\delta_{\ell}}, \quad \lambda_{\ell}=\frac{1}{\sqrt{2}} \frac{1}{3 c_{\ell}} \xi_{\ell} .
$$

If a special condition $\delta_{\ell}=-\rho_{\ell}$ is taken, one can obtain familiar relation $\left|L_{21}\right| \simeq$ $\sin \theta_{\ell} \simeq \sqrt{\left|m_{\ell 1} / m_{\ell 2}\right|}$, which was used in refs. 114 and 15. However, this condition is not guaranteed in the framework of the model. In our following analyses, a relation between $\delta_{\ell}$ and $\rho_{\ell}$ is given only by the mass $m_{\ell 1}=m_{\ell}$ as seen in eq.(4), and so the value of $L_{21}$ is given arbitrary. The $L_{13}$ and $L_{31}$ mixings are suppressed compared with $L_{12}$ and $L_{21}$ because $\lambda_{\ell}$ is $\mathcal{O}\left(m_{\mu} / m_{\tau}\right)$. On the other hand, the $L_{23}$ and $L_{32}$ mixings are almost fixed except for phases as

$$
\left|L_{23}\right| \simeq\left|L_{32}\right| \simeq \frac{1}{\sqrt{2}} \frac{m_{\mu}}{m_{\tau}} \simeq 0.04
$$

Let us turn to the neutrino sector. Assuming that the neutrinos are of the Majorana type, the neutrino mass matrix is

$$
M_{\nu}=c_{\nu}\left(\begin{array}{ccc}
1 & 0 & 0 \\
0 & 1 & 0 \\
0 & 0 & 1
\end{array}\right)+\left(\begin{array}{ccc}
\delta_{\nu} & 0 & 0 \\
0 & \rho_{\nu} & 0 \\
0 & 0 & \epsilon_{\nu}
\end{array}\right)
$$


where the first matrix is $S_{3 L}$ invariant one and the second is the symmetry breaking one. It is noted that $c_{\nu}=0$ and $c_{\nu}=\mathcal{O}(1 \mathrm{eV})$ were taken in ref. 14 and in ref. 15., respectively. Since the mass matrix of the neutrino is still diagonal one in both models, the same numerical results are obtained for flavor mixings. The neutrino masses are determined being independent of flavor mixings in this model. So the parameters $\delta_{\nu}$, $\rho_{\nu}$ and $\epsilon_{\nu}$ are easily constrained by putting $\Delta m_{21}^{2}=\Delta m_{\odot}^{2}$ and $\Delta m_{31}^{2}=\Delta m_{\text {atm }}^{2}$.

The neutrino mixing matrix $U_{\alpha i}$ is determined by only $V_{\ell}$ of the charged leptons as follows:

$$
U=V_{\ell}^{\dagger}=L^{\dagger} F^{\dagger}
$$

Then, the relevant mixing parameters of solar neutrinos are:

$$
\begin{aligned}
U_{e 1} & =\frac{1}{\sqrt{2}} L_{11}^{*}+\frac{1}{\sqrt{6}} L_{21}^{*}, \\
U_{e 2} & =-\frac{1}{\sqrt{2}} L_{11}^{*}+\frac{1}{\sqrt{6}} L_{21}^{*}, \\
U_{e 3} & =-\frac{2}{\sqrt{6}} L_{21}^{*},
\end{aligned}
$$

where $\left|L_{11}^{*}\right|^{2} \simeq 1-\left|L_{21}\right|^{2}$ and $L_{31}$ is neglected due to the suppression factor $\lambda_{\ell}$. These mixings are determined only by $L_{21}$, which is still an unknown parameter because there remains at least one undetermined parameter in the mass matrix after fixing the charged lepton masses $m_{e}, m_{\mu}$ and $m_{\tau}$ as seen in eq.(14). If the complex phases are allowed in the symmetry breaking terms, there are more unknown parameters. On the other hand, the relevant mixing parameters of atmospheric neutrinos are:

$$
U_{\mu 3}=-\frac{2}{\sqrt{6}} L_{22}^{*}+\frac{1}{\sqrt{3}} L_{32}^{*}, \quad U_{\tau 3}=-\frac{2}{\sqrt{6}} L_{23}^{*}+\frac{1}{\sqrt{3}} L_{33}^{*},
$$

where $\left|L_{22}^{*}\right|^{2}=1-\left|L_{21}\right|^{2}-\left|L_{23}\right|^{2}$ and $\left|L_{33}^{*}\right|^{2} \simeq 1-\left|L_{32}\right|^{2}$. Thus, these mixings are determined by $L_{21}$ and $L_{32}$.

As seen in eq.(11), there is a correlation between $4\left|U_{e 1} U_{e 2}^{*}\right|^{2}$ and $\left|U_{e 3}\right|$, both are relevant quantities for the oscillation probability of solar neutrinos as follows:

$$
P\left(\nu_{e} \rightarrow \nu_{e}\right) \simeq 1-4\left|U_{e 1} U_{e 2}^{*}\right|^{2} \sin ^{2} \frac{\Delta m_{21}^{2} L}{4 E}-2\left|U_{e 3}\right|^{2}\left(1-\left|U_{e 3}\right|^{2}\right)
$$


Since those are given in terms of $L_{21}=\left|L_{21}\right| \exp (i p)$, we can present allowed region on the $\left(\left|U_{e 3}\right|, 4\left|U_{e 1} U_{e 2}^{*}\right|^{2}\right)$ plane by changing $\left|L_{21}\right|$ and the phase $p$. In Fig.1, the allowed region is shown between two curves for the case of $p=0^{\circ}$ and $p=90^{\circ}$. If $\Delta m_{31}^{2} \geq 9 \times 10^{-4} \mathrm{eV}^{2}$, the mixing $\left|U_{e 3}\right|$ is constrained by the CHOOZ experiment [16], in which the oscillation probability is expressed as

$$
P\left(\bar{\nu}_{e} \rightarrow \bar{\nu}_{e}\right) \simeq 1-4\left|U_{e 3}\right|^{2}\left(1-\left|U_{e 3}\right|^{2}\right) \sin ^{2} \frac{\Delta m_{31}^{2} L}{4 E}
$$

For example, the $\mathrm{CHOOZ}$ result gave constraints $\sin ^{2} 2 \theta_{\mathrm{CHOOZ}} \leq\{0.12,0.20,0.75\}$ for $\Delta m_{31}^{2} \simeq\{5,2,1\} \times 10^{-3} \mathrm{eV}^{2}$, which correspond to $\left|U_{e 3}\right| \leq\{0.18,0.23,0.50\}$, respectively. Thus, the constraint of $\left|U_{e 3}\right|$ is strongly depend on $\Delta m_{31}^{2}$. This fact is advantage of testing the model because the solar neutrino data determines the best fit of $\left(\left|U_{e 3}\right|\right.$, $\left.4\left|U_{e 1} U_{e 2}^{*}\right|^{2}\right)$ being independent of $\Delta m_{31}^{2}$. We show the best-fit points in the three flavor analyses in ref. 10 by four black points for each $\left|U_{e 3}\right|$. If these best-fit points are reliable, we can find in Fig. 1 that $\left|U_{e 3}\right| \simeq 0.3 \sim 0.4$ is favored in the present model, which means $\Delta m_{31}^{2} \leq 10^{-3} \mathrm{eV}^{2}$ in the CHOOZ data. LBL experiments can test this prediction in the future.

However, it is important to comment on the allowed region at 95\% C.L. in ref. [10], which completely covers our predicted one. Thus, the prediction of $\left|U_{e 3}\right|$ in the model is less predictive at the present. Moreover, for $\left|U_{e 3}\right|=0.0,0.2$ and 0.4 , the values of $\chi_{\min }^{2}$ are 3.6, 4.2 and 5.6, respectively. This means that smaller values of $\left|U_{e 3}\right|$ is prefered. Therefore, we need three flavor analyses of the solar neutrino oscillation including new data of energy spectrum in Super-Kamiokande [6] [7].

LBL experiments provide an important test of the model because $\left|U_{e 3}\right|$ is a key ingredient for the $\nu_{\mu} \rightarrow \nu_{e}$ oscillation as follows:

$$
P\left(\nu_{\mu} \rightarrow \nu_{e}\right) \simeq 4\left|U_{e 3} U_{\mu 3}^{*}\right|^{2} \sin ^{2} \frac{\Delta m_{31}^{2} L}{4 E} \simeq \frac{8}{3}\left|U_{e 3}\right|^{2} \sin ^{2} \frac{\Delta m_{31}^{2} L}{4 E}
$$

where $\left|U_{\mu 3}\right|=2 / \sqrt{6}$ is put and the small $C P$ violating term is neglected. Then, the 
expected maximal oscillation probability is 0.06 for $\Delta m_{31}^{2} \simeq 5 \times 10^{-3} \mathrm{eV}^{2}$ at $\mathrm{K} 2 \mathrm{~K}$ [23 due to the constraint of the $\mathrm{CHOOZ}$ result [16].

Let us consider the case of the atmospheric neutrino. By using $L_{32} \simeq 0.04 \exp (i q)$, we can estimate $4\left|U_{\mu 3} U_{\tau 3}^{*}\right|^{2}$ as a function of $\left|U_{e 3}\right|$, where $q=0^{\circ} \sim 180^{\circ}$ and $L_{23}$ is

determined by unitarity. We present allowed region on the $\left(\left|U_{e 3}\right|, 4\left|U_{\mu 3} U_{\tau 3}^{*}\right|^{2}\right)$ plane in Fig.2, where the allowed region is shown between two curves for the case of the phase $q=0^{\circ}$ and $q=180^{\circ}$. It is found that $4\left|U_{\mu 3} U_{\tau 3}^{*}\right|^{2}$ could deviates significantly from $8 / 9$, which was presented in refs. [14] [15], due to the symmetry breaking terms in the model. The LBL experiments of $\nu_{\mu} \rightarrow \nu_{\tau}$ and $\nu_{\mu} \rightarrow \nu_{e}$ enable to test our predictions in the future.

The summary is given as follows. The mass matrix model based on the democratic form has been investigated focusing on the recent data of solar neutrinos and atmospheric neutrinos. If the solar neutrino deficit is due to the vacuum oscillation as suggested by recent Super-Kamiokande data, nearly bi-maximal mixings are needed in order to explain both solar and atmospheric neutrino deficit. In the present model, those large mixings are derived from the charged lepton sector while the neutrino mass matrix is diagonal one. It is remarked that the symmetry breaking term of the charged lepton mass matrix is very important to predict mixing angles. The model will be tested by the precise determination of the mixings and $\Delta m^{2}$ in the solar neutrinos, atmospheric neutrinos and LBL experiments in the near future.

I thank A. Smirnov for the quantitative discussion of vacuum oscillations of solar neutrinos. I also thank Y. Koide for the discussion of the model. This research is supported by the Grant-in-Aid for Science Research, Ministry of Education, Science and Culture, Japan(No.10140218, No.10640274). 


\section{References}

[1] K. S. Hirata et al., Phys. Lett. B205 (1988) 416; B280 (1992) 146;

D. Casper et al., Phys. Rev. Lett. 66 (1991) 2561;

R. Becker-Szendy et al., Phys. Rev. D46 (1992) 3720.

[2] NUSEX Collaboration, M. Aglietta et al., Europhys. Lett. 15 (1991) 559;

SOUDAN2 Collaboration, W. W. M. Allison et al., Phys. Lett. B391 (1997) 491;

Fréjus Collaboration, K. Daum, Z. Phys. C66 (1995) 417;

MACRO Collaboration, S. Ahlen et al., Phys. Lett. B357 (1995) 481.

[3] Y. Fukuda et al., Phys. Lett. B335 (1994) 237.

[4] GALLEX Collaboration, Phys. Lett. B327 (1994) 377; B388 (1996) 384;

SAGE Collaboration, J. N. Abdurashitov et al., Phys. Lett. B328 (1994) 234;

Homestake Collaboration, Nucl. Phys. B38 (Proc. Suppl.) (1995) 47;

Kamiokande Collaboration, Nucl. Phys. B38 (Proc. Suppl.) (1995) 55;

Super-Kamiokande Collaboration, Y. Fukuda et al, hep-ex/9805021 (1998).

[5] Super-Kamiokande Collaboration, Y. Fukuda et al, Phys. Rev. Lett. 81 (1998) 1562 .

[6] Y. Suzuki, invited talk at Neutrino 98, 4 - 9 June, 1998, Takayama, Japan.

[7] J.N. Bahcall, P.I. Krastev and A.Yu. Smirnov, hep-ph/9807216.

[8] A.J. Baltz, A.S. Goldhaber and M. Goldhaber, hep-ph/9806540.

[9] S.P. Mikheyev and A.Yu. Smirnov, IC/97/113, hep-ph/9708403;

B. Faïd, G.L. Fogli, E. Lisi and M. Montanino, BARI-TH/299-98, hep-ph/9805293.

[10] P. Osland and G. Vigdel, CERN-TH/98-200, hep-ph/9806339. 
[11] V. Barger, S. Pakvasa, T.J. Weiler and K. Whisnant, MADPH-98-1063, hep-ph/9806387 v3.

[12] M. Jeżabek and Y. Sumino, hep-ph/9807310.

[13] Y. Nomura and T. Yanagida, hep-ph/9807325.

[14] H. Fritzsch and Z. Xing, Phys. Lett. B 372, 265 (1996); hep-ph/9807237;

Z. Xing, DPNU-98-12, hep-ph/9804433.

[15] M. Fukugita, M. Tanimoto and T. Yanagida, Phys. Rev. D57 (1998) 4429.

[16] The CHOOZ Collaboration, M. Apollonio et al., Phys. Lett. B420 (1998) 397.

[17] Y. Suzuki, Proc. of Neutrino 96, Helsinki, June 1996, edited by K. Enqvist et al., p.237 (World Scientific, Singapore, 1997).

[18] S. G. Wojcicki, Proc. of Neutrino 96, Helsinki, June 1996, edited by K. Enqvist et al., p.231 (World Scientific, Singapore, 1997).

[19] ICARUS Collaboration, P. Cennini et al., LNGS-94/99-I, May 1994;

F. Pietropaola, invited talk at Neutrino 98, 4 - 9 June, 1998, Takayama, Japan.

[20] LSND Collaboration, C. Athanassopoulos et al., Phys. Rev. Lett. 75 (1995) 2650;

77(1996)3082; Phys. Rev. C54(1996) 2685; nucl-ex/9706006(1997);

J. E. Hill, Phys. Rev. Lett. 75 (1995) 2654.

[21] KARMEN Collaboration, Nucl. Phys. B38 (Proc. Suppl.) (1995) 235;

B. Zeitnitz, invited talk at Neutrino 98, 4 - 9 June, 1998, Takayama, Japan.

[22] Y. Koide, Phys. Rev. D 28, 252(1983); 39, 1391 (1989).

[23] M. Tanimoto, hep-ph/9806375, to be published in Phys. Lett. B. 


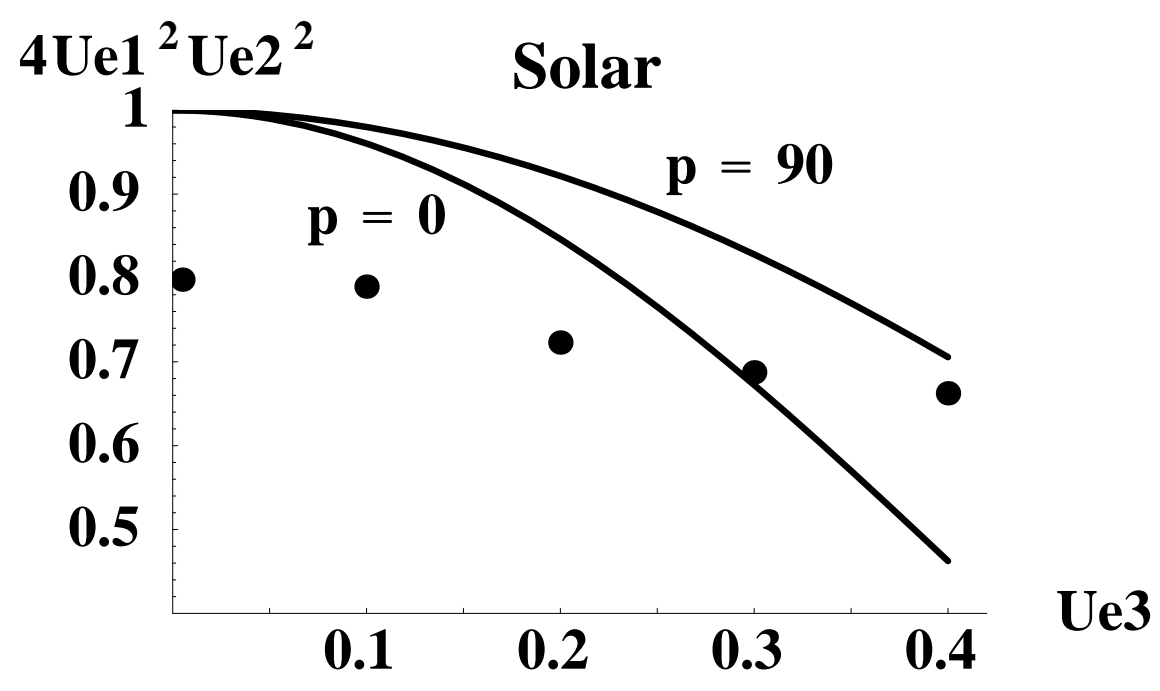

Fig. 1: Allowed region on $\left(\left|U_{e 3}\right|, 4\left|U_{e 1} U_{e 2}^{*}\right|^{2}\right)$ plane, which is the one between two curves for $p=0^{\circ}$ and $p=90^{\circ}$. Black points denote the best fits of solar neutrinos in the three flavor analyses in ref.[10] for each $\left|U_{e 3}\right|$. For $\left|U_{e 3}\right|=0.0,0.2$ and 0.4 , the values of $\chi_{\min }^{2}$ are 3.6, 4.2 and 5.6, respectively. The allowed region at 95\% C.L. completely covers our predicted one. 


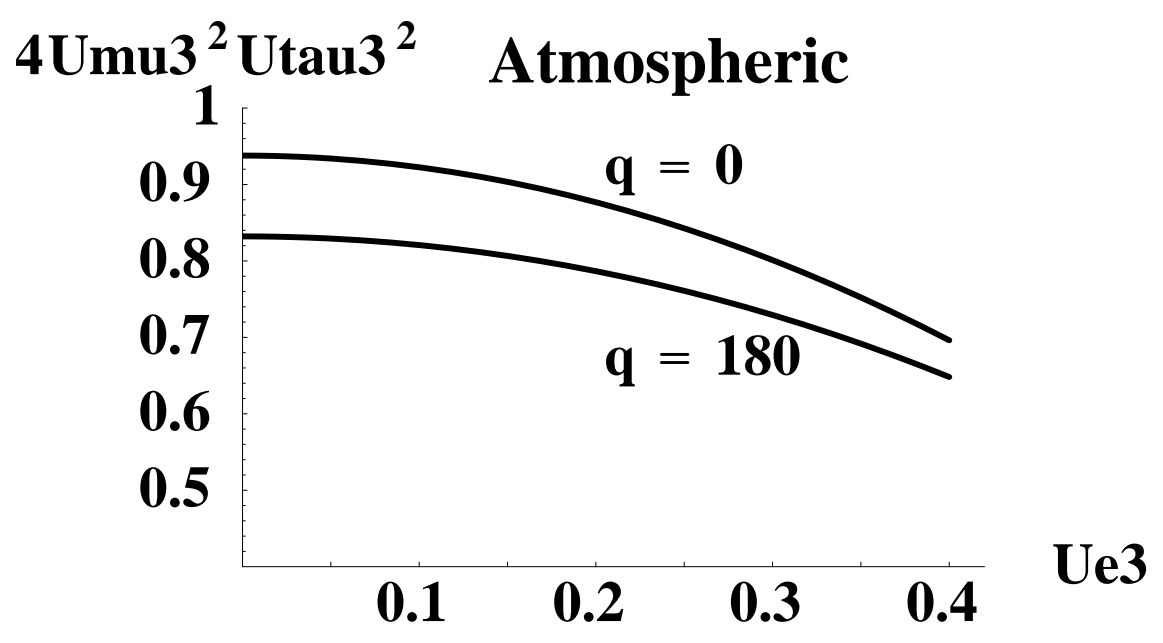

Fig. 2: Allowed region on the $\left(\left|U_{e 3}\right|, 4\left|U_{\mu 3} U_{\tau 3}^{*}\right|^{2}\right)$ plane which is the one between two curves for $q=0^{\circ}$ and $q=180^{\circ}$. 